\title{
Features of the Value-at-risk Methodology for Evaluation of Business Risks at Nonlinear Dynamics in Economic Development
}

\author{
Danchuk M. ${ }^{1}$, Kravchuk A. ${ }^{1}$, Danchuk V. ${ }^{1}$ \\ ${ }^{1}$ National Transport University, 1 Suvorov Street, Kyiv, 01010 Ukraine
}

\begin{abstract}
The novel method for business risks evaluation based on the Value-at-Risk (VaR) methodology is proposed. This method can be applicable for stable markets analysis and for description of nonlinear dynamics in economics including crisis-related economical changes. This method is driven by computer simulation of the business evolution in according with synergetic model of generalized Lorenz system.It allows VaR estimation taking into account the asymmetry and fat tail risk curve in the real time without using historical data. We used proposed method to evaluate business risks to predict possible losses as well as potential gain in the enterprise revenue.
\end{abstract}

Keywords: Value-at-Risk methodology, business risks, markets analysis, synergetic model, Lorenz system.

\section{Introduction}

The generally accepted methodology of risk evaluation that was developed by financial institutions of western economic system and the regulatory bodies (The Group of Thirty (G30 Group 1993), The Bank for International Settlements (BIS 1994), etc.) is a Value-at-Risk approach (VaR) [1]. However, this VaR methodology can only be applicable for stable markets evaluation. This methodology does not adequately reflect the value of risk when markets undergoing rapid and (or) abrupt changes under crisis-considered conditions. Moreover, the model curves of probability distribution of financial and economic indicators changes used in most existing relevant $\mathrm{VaR}$ evaluation methods, are not always appropriately describe the realities of business in modern nonlinear economy dynamics. For instance, it is well known [2] that, such distribution curves are asymmetrical and leptokurtic (sharp peaks and heavy tails). However, normal distribution curves (for the Risk MetricsTM method) are symmetrical with zero kurtosis. Pareto-Levy distribution curves (considered under the hypothesis of fractal market [3] or in most nonlinear stochastic models GARCH [3]) are leptokurtic but they have no asymmetry.

\section{Results and Discussion}

We proposed the novel method based on VaR methodology to evaluate business risks. This method can be applicable to describe the stable markets, and also to describe nonlinear dynamic economic processes. Such economic processes include fleeting (even abrupt economic changes and crises). In this paper, computer simulation of the time series of the expected revenue evolution is 
considered as a result of enterprise business in nonlinear stochastic market environment. Calculations were carried out in according with the generalized Lorenz system [3]:

$$
\left\{\begin{array}{l}
\dot{I}=I+F+\xi(t) \\
\delta \cdot \dot{F}=-F+I \cdot p+\xi(t) \\
h \cdot \dot{p}=\left(p_{e}-p\right)-I \cdot F+\xi(t)
\end{array}\right.
$$

Here $I$ - the revenue of the company at the moment $t$ as the function of the demand $Q$ for goods (services) which offered by enterprise on the market.

$F \quad-$ the function of enterprise production which determines the efficiency of conversion costs of various types of resources into the relevant products.

$p$ - the conditional price of goods (services), which generally represents a complex function which depends on multiple external and internal factors of nonlinear market environment [3].

Accordingly, $\dot{I}, \dot{F}, \dot{p}$ - represent the rate of change (time derivatives) and $\delta$, $h$ - system parameters, that define the velocity of the changes $I, F, p$ under the effect of deterministic (sudden or permanent) $p_{e}$ and stochastic $\xi(t)$ factors of the nonlinear market environment. It was assumed that $\xi(t)$ changes depend on the normal distribution and this parameter affects dynamic variables $I, F, p$ equally [3]. Modeling of corresponding time series was carried out by computer simulation method using MathCAD software taking in account the following initial values of the dynamic variables and parameters of the system (in arbitrary units): $I_{0}=0.001, F_{0}=0.01, p_{0}=0.01, \delta=5$, $h=2.5$., observation time $\Delta t=150$. We selected values $p_{e}$ that corresponded to the transitions of the system (1) from a point of bifurcation to the stationary fixed state and the deterministic chaos state (i.e. economic crisis). We used obtained time trends data to determine the parameters of probability distribution curves of the expected revenue $I$ in according with [3], namely the skewness coefficient $\beta$ and kurtosis $\theta$. We analyzed probability distribution curves for expected revenue in case of nonlinear deterministic trend of the company activity evolution (i.e. during the transition from a fixed point bifurcation to the equilibrium state $(D=0))$ and we found that these curves are characterized by asymmetry $(\beta \neq 0)$ and they have leptokurtosis $(\theta>0)$. Interestingly, we observed disappearance of asymmetry and leptokurtosis on probability distribution curves $I$ when we taken in account the additional effect of stochastic nonlinear market environment factors $(D \neq 0)$ on the financial and economic activity of the enterprise. The results of computer simulation of the company evolution at the system (1) transiting from the bifurcation point to the state of chaos (economic crisis, $p_{e}=37$ ) show that probability distribution curves for expected revenue are characterized by small values of asymmetry and leptokurtosis.

To generate histograms showing a frequency distribution of expected revenue $I$ we used numerical data obtained in the analysis of time series for system (1) and its elements were taken as follows:

$$
x_{i}=I_{t+1}-I_{t}, \quad i=\overline{1, n-1}
$$

Density plots of normalized frequency distribution of expected revenue were built using standard functions in MathCAD for different time series (2). These time series were generated by 
computer simulation of the system evolution (1) taking into account different values of deterministic and stochastic nonlinear factors of the market environment. This normalization was performed per unit at maximum density. To be specific, the number of histogram distribution segments was chosen equal to ten in all cases. For example, Figure 1 shows the histograms built for $p_{e}=5$ and $D=0$ (Fig. 1a) and $p_{e}=5$ and $D=5$ (Fig. 1b).

The next step was required to determine the quantitative characteristics of business risk. This value was obtained by plotting of approximate function describing the distribution density of enterprise expected revenue $P(x)$ which fitted the best into these distribution histograms. In general, the distribution curves could be asymmetric. Therefore, the approximate function is proposed as follows:

$$
P(x)=a_{1}^{-} \cdot e^{-a_{2}^{-}\left(\left|x-a_{3}^{-}\right|\right)^{a_{4}^{-}}}+a_{1}^{+} \cdot e^{-a_{2}^{+}\left(\left|x-a_{3}^{+}\right|\right)^{a_{4}^{+}}},
$$

where $a_{1}^{-}, a_{2}^{-}, a_{3}^{-}, a_{4}^{-}, a_{1}^{+}, a_{2}^{+}, a_{3}^{+}, a_{4}^{+}$

- the approximation parameters for the left hand (-) and for the right side (+) of the distribution curves, respectively. We postulate that $a_{3}^{-}=a_{3}^{+}$in (3) and these values are correspond to the enterprise expected revenue with maximum probability.

This analysis shows that there is a good agreement between the results of numerical approximations and corresponding histograms in all cases. Next, we performed a renormalization of the density function of probability distribution of expected revenue $P(x)$ per unit area under the curve in the form of
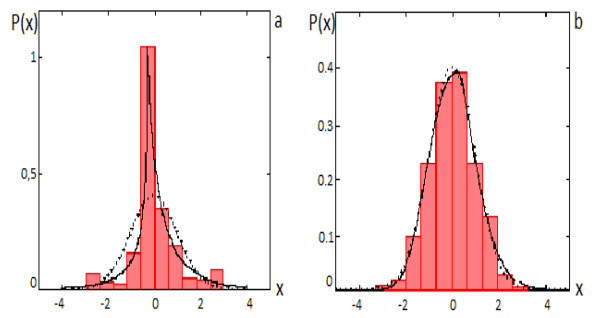

Fig. 1: Density function of the normalized probability distributions of expected revenue as the results of simulation time trends of company evolution in system (1). Histograms - normalized frequency distribution of expected revenue for time series (2), solid line - approximate functions according to (3); dashed line normal (Gaussian) distribution; a for $p_{e}=5$ and $D=0 ; \mathrm{b}$ - for $p_{e}=5$ and $D=5$.

$$
\widetilde{P}(x)=\frac{P(x)}{\int_{a}^{b} P(x) d x},
$$

where $\mathrm{a}, \mathrm{b}$ - corresponding integration limits, and then we calculated the cumulative function of probability distribution of expected revenue using the following formula:

$$
F(x)=\int_{a}^{x} \tilde{P}(x) d x .
$$

Fig.2 shows an example of functions $\tilde{P}(x)$ and $F(x)$ for time series trend changes in expected revenue for $p_{e}=5$ and $D=0.1$.

Thus, the described method allows the adequate estimation of $\mathrm{VaR}$ value taking into account the asymmetry and fat tail risk curve in the real time without using historical data. We propose to use this novel approach to evaluate business risks within the frameworks VaR methodology. 
This method based on an analysis of the distribution curve (4) and (5) obtained in simulation studies of time trends in the evolution of system (1), and it allows to perform evaluation of $\mathrm{VaR}$ values for potential losses $\left(\mathrm{VaR}_{-}\right)$as well as the possible gain in revenue $\left(V a R_{+}\right)$. In addition, in some cases quantitative comparison $\mathrm{VaR}_{-}$and $\mathrm{VaR}_{+}$could generate the additional information for managers to make the most optimal management decisions related to enterprise financial and economic activities.

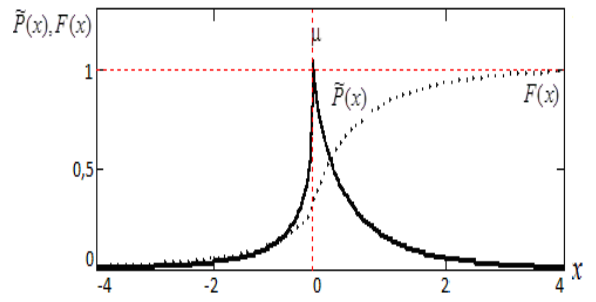

Fig.2:Probability distribution of enterprise expected revenue $I$ derived from computer simulation of time trends of the company evolution in according with model system (1), normalized per unit area (for $p_{e}=5$ and $D=0.1$ ).

Solid line - density distribution function $\widetilde{P}(x)$; dashed line - the cumulative distribution function $F(x)$.

According to mentioned above, the maximum possible value of expected cash loss in the revenue with confiding probability level $(1-\alpha)$ at the certain time horizon of the forecast $\operatorname{VaR}_{-}^{(1-\alpha)}$ (see Fig. 2) is defined as follows:

$$
\operatorname{VaR}_{-}^{(1-\alpha)}=F(\alpha)=\int_{a}^{\alpha} \tilde{P}(x) d x,
$$

where $F(\alpha)$ - quantile of level $\alpha$ for cumulative function of probability expected revenue according to (5).

Thus, the value of the maximum possible expected gain in the revenue with the confiding probability level $(1-\alpha)$ at the same time horizon of the forecast $\mathrm{VaR}_{+}^{(1-\alpha)}$ (see Fig. 2) is equal to quantile of level $(1-\alpha)$ for cumulative function of probability expected revenue $F(1-\alpha)$ according to (5) and was calculated as follows:

$$
\operatorname{VaR}_{+}^{(1-\alpha)}=F(1-\alpha)=\int_{a}^{1-\alpha} \widetilde{P}(x) d x
$$

We propose to use the semi-variance method and the value of semi-standard deviation from mode (as the quantitative risk measures) to estimate absolute values of the risk of losses and earnings which resulted from the proper financial and economic activity of enterprise.

\section{References}

[1] J. Mina, J. Xiao, "Return to RiskMetrics: The evolution of a standard" RiskMetrics Group, New York, 111 p., 2001.

[2] E. Peters "Fractal Market Analysis: applying chaos theory to investment and economics", John Wiley \& Sons, 336 p., 1994.

[3] M.V. Danchuk, L.S. Kozak, A.P. Kravchuk "Study of synergistic nature of the business risk curve the mechanisms", Visnyk NTU (Ukraine), pp. $383-395,2012$. 